\title{
Nota científica \\ ANATOMÍA DEL OVARIO DE LA YEGUA MEDIANTE SECCIONES PLASTINADAS CON EL MÉTODO E12
}

\author{
Scientific note
}

ANATOMY OF THE MARE OVARY USING PLASTINATED SECTIONS BY THE METHOD E12

\author{
Horst Erich König ${ }^{1 *}$, Mircea-Constantin Sora ${ }^{2}$, Johannes Seeger ${ }^{3}$, y Sergio Donoso ${ }^{4}$
}

${ }^{1}$ Institut für Anatomie, Histologie und Embryologie, der Veterinärmedizinischen Universität Wien, Veterinärplatz 1, A-1210 Viena, Austria.

2 Zentrum für Anatomie und Molekulare Medizin Sigmund Freud Privatuniversität Wien, Freudplatz 1, 1020 Wien, Austria

${ }^{3}$ Veterinär-Anatomischen Institut, der Universität Leipzig, An den Tierkliniken 4304103 Leipzig, Alemania.

${ }^{4}$ Departamento de Patología y Medicina Preventiva, Facultad de Ciencias Veterinarias, Universidad de Concepción, Casilla 537, Chillán, Chile.

* Autor para correspondencia E-mail: Horst.Koenig@vetmeduni.ac.at

\section{RESUMEN}

La estructura macroscópica del ovario de la yegua es diferente al de otros mamíferos domésticos, debido a que la zona parenquimatosa se ubica en el centro del ovario. La zona parenquimatosa está rodeada por la zona vascular, la que adquiere forma de campana. El ovario de la yegua es mucho más grande que otras especies, en relación al tamaño corporal, como por ejemplo los bovinos. El objetivo de este estudio fue demostrar la estructura especial del ovario de la yegua, realizando secciones translúcidas del ovario mediante el método E12. Para ello se colectaron 14 ovarios de yegua, seleccionados mediante palpación, escogiendo dos ovarios con estructuras funcionales (folículos y cuerpos luteos). Los ovarios se cortaron y se sometieron al proceso de plastinación mediante el método E12 y luego se cortaron en láminas translúcidas de $2 \mathrm{~mm}$. En un ovario se encontró un folículo maduro en el cual se podía ver el estigma. En el segundo ovario se encontraron múltiples folículos y un cuerpo luteo. Los cortes obtenidos sirven como material de estudio para médicos veterinarios y estudiantes e ilustran lo que se observa en los equipos de ultrasonido.

Palabras clave: anatomía, ovario, folículo, cuerpo lúteo, yegua, plastinación, cortes E12.

\section{ABSTRACT}

The macro-anatomical organization of the mare ovary differs from other mammalians since the parenchymal zone is positioned inside the ovary. The parenchymal zone is bell-shaped and it is surrounded by the vascular zone. Regarding body size, the ovary of mares is bigger compared to that of other species, such as cows. The objective of this study was to verify the special structure of a mare ovary by obtaining transparent slices using the E12 method. A number of 14 mare ovaries were collected and 2 of them were selected through palpation based on the presence of functional structures (follicles and a corpus luteum). These specimens were plastinated using the E12 method and cut into 2-mm transparent slices. A mature follicle was found in one of the ovaries, and the stigma could 
also be seen. Several follicles and a corpus luteum were found on the slices of the other ovary. These slices can be used as a teaching/learning resource by clinicians and students since the structures observed are similar to those obtained using ultrasound.

Key words: anatomy, ovary, mare, plastination, E12 sections.

\section{INTRODUCCIÓN}

La plastinación es considerado el mejor método para la preservación de tejido animal perecible, para el estudio de la anatomía de diversas especies (Sora y Cook, 2007).

El ovario de la yegua, en comparación con el de otros mamíferos domésticos, tiene la zona vascular en la superficie del órgano. Esa zona rodea como una campana el ovario en el margen mesovarial. En la parte opuesta del ovario queda una zona libre de vasos sanguíneos mayores, la fosa ovárica (fossa ovarii), que está cubierta por un epitelio germinativo (Liebich, 2010). En la fosa ovárica, el folículo maduro (folículo de Graaf) alcanza la superficie del ovario. En este lugar se desarrolla el estigma, que es donde tiene lugar la ovulación y se forma el cuerpo luteo (König et al., 2015).

El diagnóstico ovulatorio transrectal, por la estructura anatómica del ovario de la yegua, no es tan fácil como en la vaca, donde los folículos y los cuerpos luteos se ubican en la zona parenquimatosa, en la superficie del órgano. Por este motivo, actualmente, para este diagnóstico en la yegua se usa el metodo de ultrasonido (Aurich, 2009; Gastal, 2011), en donde se aprovecha la diferencia de densidad entre el líquido folicular y la teca del folículo, lo que entrega una imagen virtual, a diferencia de las secciones plastinadas, las cuales entregan una imagen real de la forma y de la estructura del folículo y del cuerpo luteo. Estos secciones son secas, sin olor, no tóxicas y se pueden conservar en forma permanente. Se pueden usar en la enseñanza de estudiantes de medicina veterinaria, en la clínica y en la anatomía. Se pueden mostrar también al dueño de los animales en el cual se hizo el diagnóstico en el ovario.

Existen diversas técnicas de plastinación, entre las cuales destaca la E12. Esta técnica consiste en que a partir de tejido animal fresco, se hacen láminas o cortes transparentes de un grosor de 2 a $5 \mathrm{~mm}$, que permiten visualizar tanto estructuras macroscópicas como submacroscópicas, evidenciando detalles de la posición y estructura anatómica tanto normal como patológica de diversos órganos (Sora y Cook, 2007).

Este trabajo pretende entregar mayores antecedentes sobre la anatomía seccional del ovario de yegua con el método de plastinacion E12 (Sora y Cook, 2007), dado que existe muy poca información.

\section{MATERIALES Y MÉTODOS}

En un matadero para equinos ubicado en la ciudad de Freital, cerca de Leipzig, Alemania, se recolectaron 14 ovarios de yeguas. Se conocía la edad de los animales pero no la etapa hormonal. Los ovarios se examinaron macroscópicamente. Mediante palpación se localizaron los folículos, eligiendo dos ovarios izquierdos, uno de una yegua de 8 años y otro de una yegua de 11 años. En la palpacion de los órganos no fue posible hacer la diferenciación entre folículos y cuerpos luteos.

Para hacer los cortes E12, se empleó el siguiente método: los ovarios se fijaron durante 8 días en una solución de formalina al 4\%, a una temperatura de $5^{\circ} \mathrm{C}$. Despues se enfriaron por 5 días a una temperatura de $45^{\circ} \mathrm{C}$ bajo cero. Esta temperatura de congelación previene la formación de microcristales de hielo en el tejido. Los órganos enfriados se seccionaron en cortes con un grosor aproximado de 2 milímetros. Después los cortes se deshidrataron en baños de acetona a una temperatura de $25^{\circ} \mathrm{C}$ bajo cero. Los cortes obtenidos se procesaron a continuación según la técnica de plastinación estándard E12, descrita por An y Zhang (1999), Lane (1990), y Sora y Cook (1999). Finalmente, los cortes se impregnaron con la resina E12, y se secaron a una temperatura de $45^{\circ} \mathrm{C}$.

\section{RESULTADOS}

En una de las secciones del ovario de la yegua de 8 años se encontró un folículo maduro (de Graaf) poco antes de la ovulación. Cerca de la fosa ovárica, la pared del folículo era mucho más delgada. Las tecas del folículo, interna y externa, no presentaron pliegues. En el mismo ovario no se encontró ningun cuerpo luteo.

En el ovario de la yegua de 11 años se encontraron muchos folículos de diferente tamaño. En el mismo ovario se encontró un cuerpo luteo de una generacion anterior. En los tres cortes (Fig. 1, 2 y 3) se pueden ver los cuerpos ováricos funcionales, folículos y cuerpo luteo, al igual que en las imágenes de ultrasonido.

\section{DISCUSIÓN}

Existen muchas publicaciones sobre cuerpos funcionales de los ovarios (folículos y cuerpos luteos) de los rumiantes y carnívoros (König et al., 


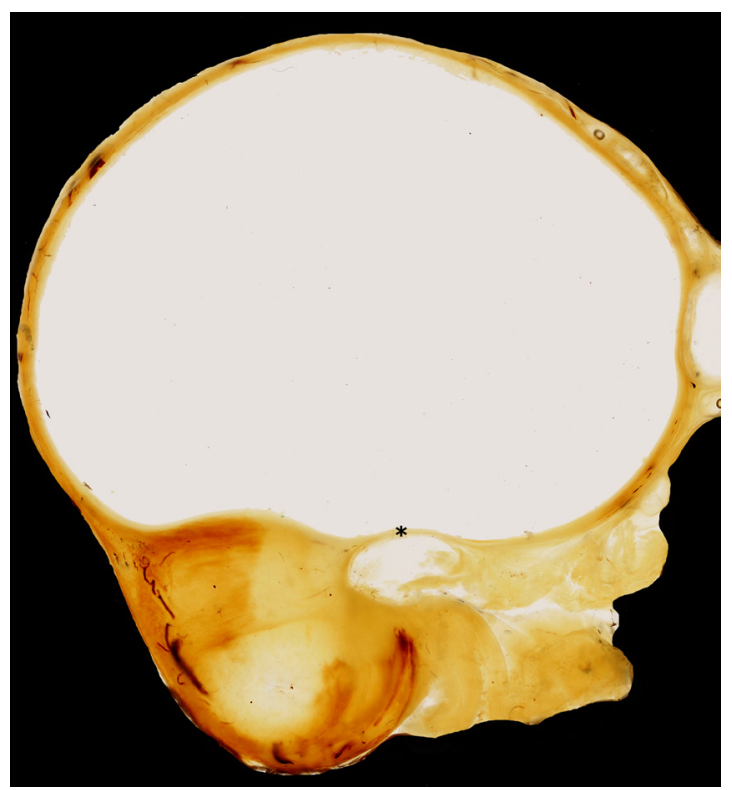

Fig. 1. Corte plastinado E12 del ovario de una yegua de 8 años; se observa el folículo maduro de Graaf y el estigma*.

Fig. 1. E12 slice of the ovary from an 8-year old mare with Graafian follicle. The stigma* is visible

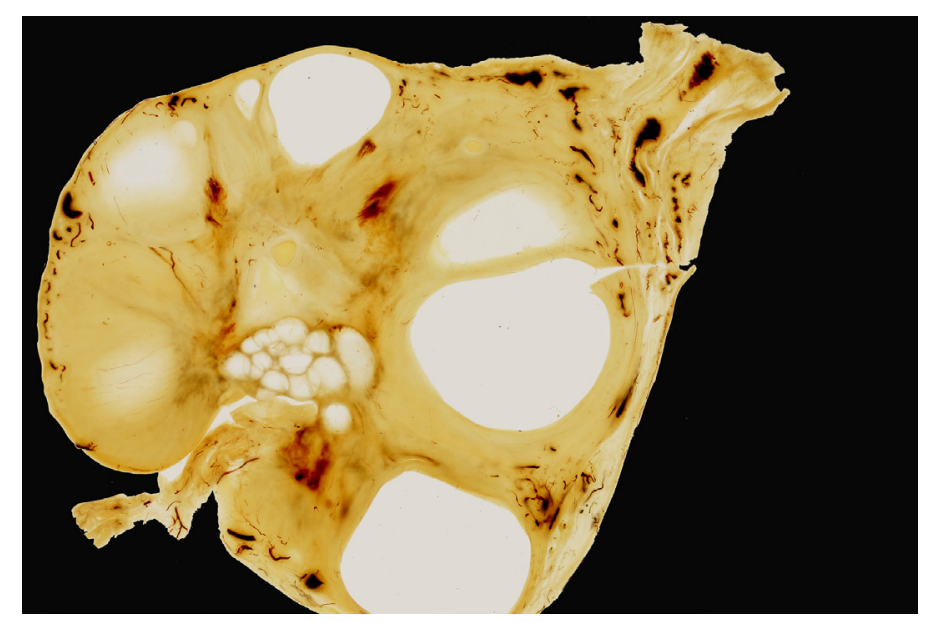

Fig. 2. Corte plastinado E12 del ovario de una yegua de 11 años donde se observan múltiples folículos. Fig. 2. E12 slice of the ovary from an 11-year old mare with many follicles.

1987; Rüsse y Sinowatz, 1991), junto con investigaciones sobre los vasos sanguíneos del ovario de la yegua mediante la técnica de inyecciones con metilmetacrilato (König y Ries 1987; König, 1995). Sin embargo, hasta ahora no hay publicaciones sobre el ovario de la yegua mediante la utilización de secciones plastinadas transparentes con la técnica E12. Sobre todo faltan datos sobre la forma, tamaño y la ubicacion de los cuerpos funcionales (folículos y cuerpos luteos) en secciones plastinadas con la técnica E12.

En la yegua es muy importante precisar exactamente el estadio del ciclo ovulatorio, para elegir el momento óptimo de la inseminación. Esto actualmente no se realiza mediante la palpación transrectal sino que con el método de ultrasonido (Aurich, 2009; Glatzel, 2009; Gastal, 2011).

Los vasos sanguíneos en el ovario juegan un rol importante en su funcionamiento. Ellos transportan los hormonas de la hipófisis al ova- 


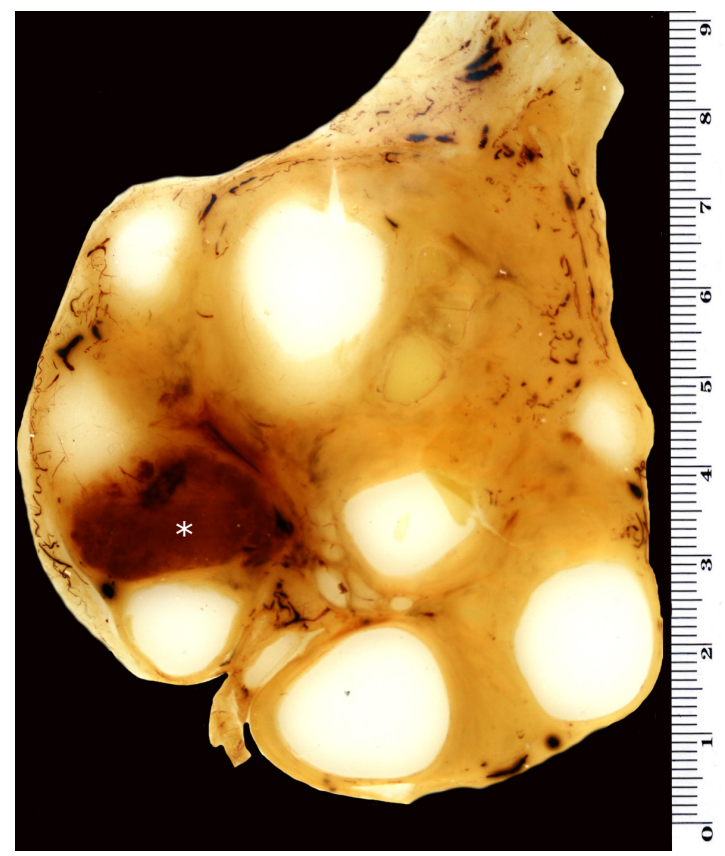

Fig. 3. Corte plastinado E12 del ovario de una yegua de 11 años, donde se observan múltiples folículos y un cuerpo luteo*.

Fig. 3. E12 slice of the ovary from an 11-year old mare with many follicles and corpus luteum*.

rio para dirigir su función. Del ovario también se transportan, por medio de la sangre, hormonas que influyen en la la función cíclica de los otros componentes del aparato genital de la hembra. Otras hormonas que influyen en el crecimiento y la luteólisis del cuerpo luteo, alcanzan el ovario también por vía sanguínea (Aurich, 2009). Otros órganos de gran importancia para el recién nacido, también se encuentran bajo la influencia de las hormonas del ovario, como por ejemplo, la glándula mamaria (Bartmann et al., 2010). Los vasos sanguíneos, de gran tamaño en el ovario de la yegua, circulan por la túnica albuginea que cubre el ovario, en comparación con los otros mamíferos domésticos (König y Ries, 1987). En la vaca y otros mamíferos domésticos, estos vasos sanguíneos quedan en el centro del ovario, es decir en la médula ovárica. Desde el centro del ovario se distribuyen hacia la periferia, para alcanzar los folículos y cuerpos luteos que se ubican en la corteza ovárica (cortex ovarii) (König et al., 1987). En todos los casos, para cada folículo y cuerpo luteo existe una arteria y una vena principal, que transporta la sangre hacia el cuerpo funcional y desde este a todo el cuerpo del animal (Hees et al., 1988; König, 1995; König et al., 2015). Con el método de corrosión (König, 1995) fue posible mostrar que alrededor del estigma, antes de la ovulacion en el ovario de la yegua, hay un borde de finos capilares, que rodean el estigma (König, 1995; König et al., 2015 ). Con técnicas de corrosión e histológicas, se encuentran capilares típicos para celulas hormonales alrededor de las células de la teca folicular, y de la celulas luteales (Hees et al., 1988). En los cortes plastinados se pueden ver muy bien los grandes vasos sanguíneos. Los capilares, que se pueden observar con el microscopio electrónico de barrido en las piezas de corrosión (Hees et al., 1988), no son visibles en los cortes plastinados.

\section{CONCLUSIONES}

Mediante la utilización del método de plastinación E12, se pueden observar los folículos y cuerpos luteos del ovario, de utilidad en la clínica veterinaria y en la enseñanza universitaria.

El método de plastinación E12 permite obtener cortes de ovario trasnslúcidos y atóxicos, en donde se puede observar hasta el estigma en el folículo maduro de Graaf.

Los cortes de ovario obtenidos mediante este método no se deterioran, lo que permite conservarlos en forma permanente.

\section{LITERATURA CITADA}

An, P.C., and M. Zhang. 1999. A technique for preserving the subarachnoid space and its contents in a natural state with different colours. J. Int. Soc. Plastination 14:12-17. 
Aurich, C. 2009. Reproduktionsmedizin beim Pferd. 2. Aufl. Parey im MVS Medizinverlag. 1-4:71-80. Stuttgart, Germany.

Bartmann, C.P., H. Übermuth, und H. Wissdorf. 2010. Weibliche Geschlechtsorgane, Milchdrüse, Euter und Harnröhre. p. 779786. In Praxisorientierte Anatomie und Propädeutik des Pferdes. H. Wissdorf, $\mathrm{H}$. Gerhards, B. Huskamp, E. Deegen (eds.). 3 ed. Verlag M. \& H. Schaper, Hannover, Germany.

Glatzel, P.S. 2009. Sonographie in der Pferdereproduktion. p. 180-183. In Atlas der Anatomie des Pferdes. Budras K.-D. y Röck, S. (eds.). Schlütersche, Hannover, Germany.

Gastal, E.L. 2011. Ovulation: Part 2. Ultrasonographic morphology of the preovulatory follicle. p. 2031-2054. In A.O. McKinnon, E.L.Squires, W.E. Vaala, and D.D. Varner (eds.) Equine reproduction. 2nd ed. Blackwell Publishing Ltd., Chichester, UK.

Hees, H., H.E. König, und I. Hees. 1988. Angioarchitektonische Untersuchungen am Blutgefäß-System der Funktionsgebilde im Ovar der Stute. Fertilität 4:30-34.

König, H.E, W. Amselgruber, und I. Rüsse. 1987. Zur Mikrozirkulation in Follikeln und Corpora lutea des Rinderovars. Eine korrosionsanatomische Studie. Tierärztl. Prax. 16:25-31.
König, H.E, und R. Ries. 1987. Korrosionsanatomische Untersuchungen am Blutgefäßsystem des Eierstockes der Stute. Tierärztl. Prax. 15:181-184.

König, H.E. 1995. Zur Anatomie des Eierstockes der Stute. Acta Vet. Brno. 64:13-16

König, H.E, J. Plendl, und H-G. Liebich. 2015. Weibliche Geschlechtsorgane (Organa genitalia feminina). In Von König, H.E. u. Liebich, H-G. (eds.). Anatomie der Haussäugetiere. p. 421-428. 6. ed. Schattauer Verlag, Stuttgart, Germany.

Lane, A. 1990. Sectional anatomy: standardized methodology. J. Int. Soc. Plastination 4:16-22.

Liebich, H-G. 2010. Funktionelle Histologie der Haussäugetiere und Vögel. p. 323-331. 5. ed. Schattauer Verlag, Stuttgart, Germany.

Rüsse, I., und F. Sinowatz. 1991. Lehrbuch der Embryologie der Haustiere. p. 74-84. Verlag Paul Parey, Berlin und Hamburg, Germany.

Sora, M.-C., und P. Cook. 2007. Epoxy plastination of biological tissue: E12 technique. J. Int. Soc. Plastination 22:40-45. 